LAWRENCE LIVERMORE N A T IO N A L LABORATORY

\title{
Edge Simulation Laboratory Progress and Plans
}

R.H. Cohen

June 6, 2007 
This document was prepared as an account of work sponsored by an agency of the United States Government. Neither the United States Government nor the University of California nor any of their employees, makes any warranty, express or implied, or assumes any legal liability or responsibility for the accuracy, completeness, or usefulness of any information, apparatus, product, or process disclosed, or represents that its use would not infringe privately owned rights. Reference herein to any specific commercial product, process, or service by trade name, trademark, manufacturer, or otherwise, does not necessarily constitute or imply its endorsement, recommendation, or favoring by the United States Government or the University of California. The views and opinions of authors expressed herein do not necessarily state or reflect those of the United States Government or the University of California, and shall not be used for advertising or product endorsement purposes.

This work was performed under the auspices of the U.S. Department of Energy by University of California, Lawrence Livermore National Laboratory under Contract W-7405-Eng-48. 


\section{Edge Simulation Laboratory \\ Progress and Plans \\ Report to PSACI June 2007; \\ presented by R. Cohen on behalf of ESL}

The Edge Simulation Laboratory (ESL) is a project to develop a gyrokinetic code for MFE edge plasmas based on continuum (Eulerian) techniques. ESL is a base-program activity of OFES, with an allied algorithm research activity funded by the OASCR base math program. ESL OFES funds directly support about 0.8 FTE of career staff at LLNL, a postdoc and a small fraction of an FTE at GA, and a graduate student at UCSD. In addition the allied OASCR program funds about $1 / 2$ FTE each in the computations directorates at LBNL and LLNL. OFES ESL funding for LLNL and UCSD began in fall 2005, while funding for GA and the math team began about a year ago.

ESL's continuum approach is a complement to the PIC-based methods of the CPES Project, and was selected (1) because of concerns about noise issues associated with PIC in the highdensity-contrast environment of the edge pedestal, (2) to be able to exploit advanced numerical methods developed for fluid codes, and (3) to build upon the successes of core continuum gyrokinetic codes such as GYRO, GS2 and GENE.

The ESL project presently has three components: TEMPEST, a full-f, full-geometry (singlenull divertor, or arbitrary-shape closed flux surfaces) code in E, $\mu$ (energy, magnetic-moment) coordinates; EGK, a simple-geometry rapid-prototype code, presently $\delta f$; and the math component, which is developing and implementing algorithms for a next-generation code. Progress would be accelerated if we could find funding for a fourth, computer science, component, which would develop software infrastructure, provide user support, and address needs for data handing and analysis. We summarize below the status and plans for the three funded activities.

\section{TEMPEST}

Tempest, a code begun under LLNL LDRD funding, is written in velocity coordinates $\mathrm{E}_{0}$ (kinetic energy plus a zero-order potential) and magnetic moment $\mu$, a choice made to facilitate accurate, low-dissipation calculation of streaming along magnetic field lines. The spatial coordinate system is field-line following with shifted-periodic toroidal boundary conditions in the closed-flux-surface region and absorbing or partially reflecting boundary conditions (with complete reflection off of a confining sheath potential for electrons). The discretization is fourth and fifth order finite-difference in space, with options for second- or fourth-order finite volume or finite difference in velocity space. The current discretization schemes are not explicitly conservative but appear to have adequate conservation properties for moderate resolution. The code presently solves a long-wavelength limit of the gyrokinetic Poisson equation. (A more general orbit-averaging procedure has been implemented but not yet coupled to the field solve.) The code includes several collision options ranging from simple Krook to callouts to an external Fokker-Planck package (CQL) to calculate a fully nonlinear collision operator. Electrons can be treated either as a (nonlinear) adiabatic (Boltzmann) species or as a second gyrokinetic species.

Development work over the past year has included implementation of the finite-volume velocity-space option and an associated collision package, porting the code from the LLNLdeveloped SAMRAI adaptive-mesh-refinement framework to the more actively (and OASCR-) 
supported CHOMBO framework, and extension of the code from 4D to 5D (from two to three spatial dimensions.)

Code applications have been primarily focused on verifying/validating the 4D version, specifically in the areas of neoclassical transport and geodesic acoustic modes (GAMs). In the neoclassical transport area, we have performed a density scan with no field solve and velocitydependent Lorentz collisions, and a plateau-regime run with Krook collisions including a twodimensional solution of the gyrokinetic Poission equation, and in both cases obtained reasonably good agreement with analytic theory. We have also performed a cross-code comparison with XGC-0 (a code from the CPES Center) of neoclassical transport for edge-like (strong) density and temperature gradients, in both an annular closed-fluxsurface geometry and in a DIII-D divertor geometry. These runs were done with Lorentz collisions and no field solve; comparisons were made of temperature and parallel velocity profiles, and better-than-factor-of-two agreement was found. The TEMPEST divertor run was repeated with reversed toroidal field; the trends in parallel velocity flow and divertor heat and particle fluxes are qualitatively consistent with experimental observations on DIII-D (and other tokamaks). The TEMPEST runs were performed with a mesh geometrically spaced in both $\mathrm{E}_{0}$ and $\mu$, which proved to be very helpful in enabling adequate resolution in regions of both high and low temperature; a more efficient solution will be available in the next-generation ESL code via spatially dependent adaptive mesh refinement of the velocity variables.

GAM simulations have been done with boundary conditions that are periodic both radially and poloidally, and with adiabatic electrons. Scans were done over safety factor $\mathrm{q}$ and inverse aspect ratio $\varepsilon$. The results for the decay rate versus $q$ are close to those obtained from the recent theoretical calculation of Sugama and Watanabe, which accounts for finite-orbit-width effects and

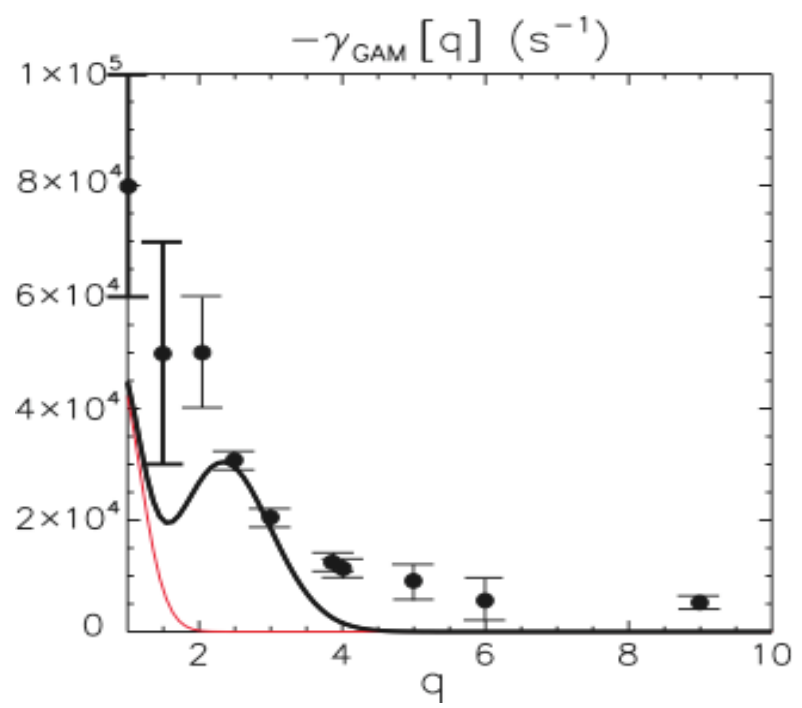

Fig.1. GAM damping rate versus $q$ for TEMPEST, compared with theory of Sugama and Watanabe (black) and 0-orbitwidth theory (red)

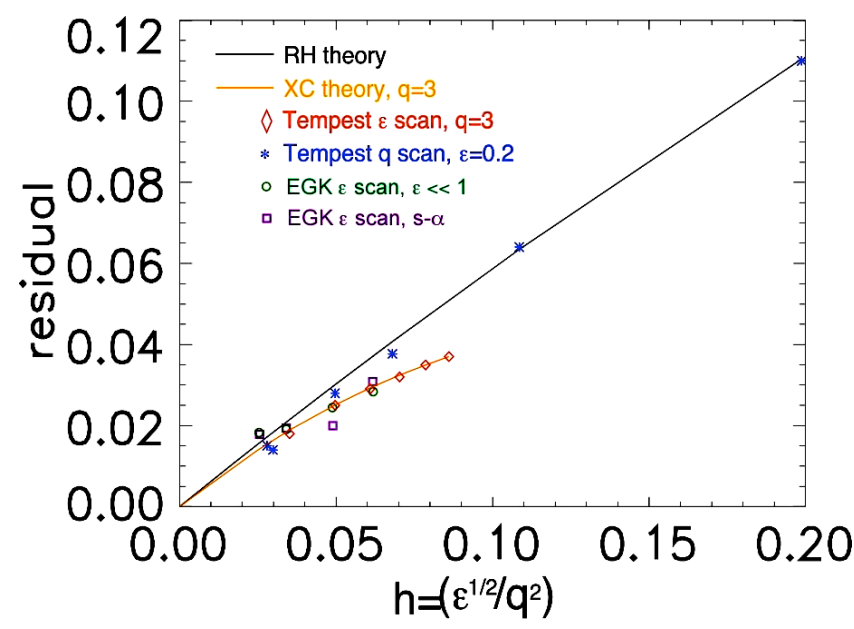

Fig. 2. GAM residual scans, TEMPEST and EGK versus theory yields a substantially larger damping rate at finite $q$ than the older zero-orbit-width result. A scan of residual amplitude versus $\varepsilon$ is in quite close agreement with the theory of Xiao and Catto, as well as with a similar scan done using the EGK code, while a scan of residual versus q agrees with the older Hinton-Rosenbluth result; see Fig. 2. 
Some preliminary testing of the 5D (3 spatial dimensions; turbulence) capabilities of the code was done in the fall, with low-resolution simulations of a stable drift wave and an iontemperature-gradient mode. We will be returning to testing of the 5D code this summer.

An option presently being debugged in the code is the inclusion of a velocity-dependent anomalous radial diffusion term. The objective is to be able to simulate effects of anomalous turbulent transport on confinement timescales (a kinetic analogue of today's 2D edge fluid transport codes). Through appropriate choices of the velocity dependence one can independently reproduce the effect of various members of the fluid transport matrix (e.g. density and thermal diffusion coefficients) when operating on a Maxwellian distribution.

\section{EGK}

EGK is a finite-difference code in $\mathrm{v}_{\|}$ $\mu$ coordinates, whose objective is rapid exploration of issues associated with gyrokinetic simulation under edge-like conditions. The present $\delta$ f code version has been subjected to a number of verification tests: (1) comparison of growth rates of trapped-electron modes and ITG modes with GS2; good agreement is obtained; (2) a scan of residual versus $\varepsilon$ for geodesic acoustic modes; the comparison with TEMPEST and with theory is shown in Fig. 2; (3) neoclassical tests, including a scan with density of the neoclassical radial fluxes for Lorentz collisions with a constant collision frequency (no electric field) as well as self-consistent $1 \mathrm{D}$ potential solutions. The code has been used to explore dissipation and possible instabilities associated with various
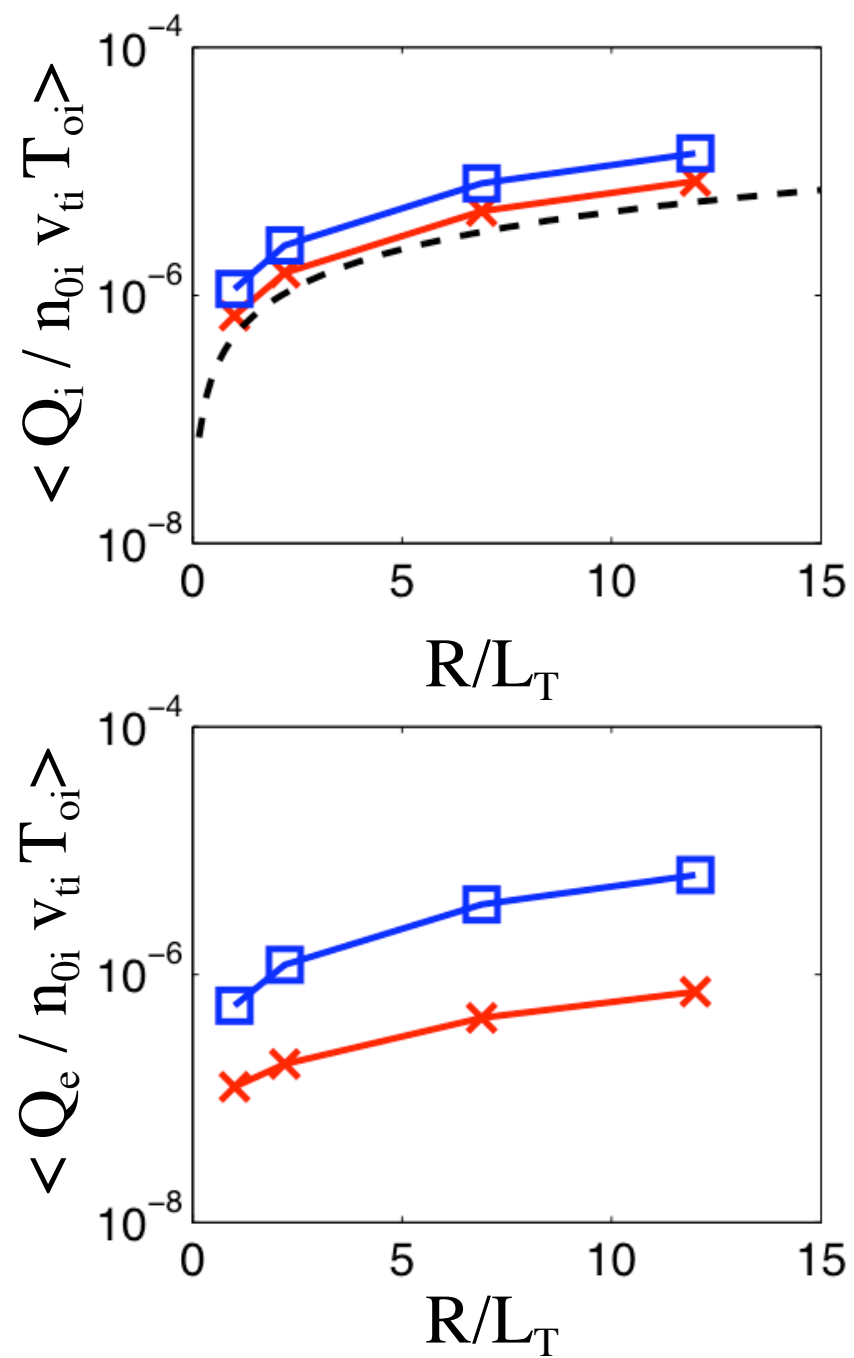

Fig. 3. Ion and electron neoclassical heat fluxes from EGK with and without poloidal dependence in potential approaches to parallel-velocity discretization (particularly in the neighborhood of the passingtrapped separatrix).

Recently the code has successfully solved the 2D gyrokinetic Poisson equation to obtain the neoclassical electric field including poloidal variation, with kinetic electrons and ions. The results show little change in ion dynamics from results with a $1 \mathrm{D}$ potential solution, but the electron heat flux is enhanced (Fig. 3). 
These tests are part of a campaign to combine neoclassical and drift-wave physics and develop a unified potential solution strategy that works in both limits.

\section{Applied Math Activity}

The applied math component is developing and implementing algorithms for fourth-order, conservative, positivity-preserving finite-volume discretization of the gyrokinetic equation on mapped multiblock grids (which can accommodate the complex topology of a diverted tokamak magnetic field). These algorithms and the infrastructure being developed to support them will form the basis for our next-generation code, which will feature the above attributes and will be written in $\mathrm{v}_{\|}-\mu$ coordinates. The new code, like TEMPEST, is being developed in an adaptivemesh-refinement (AMR) framework which provides "born parallel" capabilities and also will be exploited in the future for spatially-dependent velocity mesh refinement (and possibly spatial mesh refinement as well).

Recent progress has included (1) a demonstration that $4^{\text {th }}$-order-accurate flux divergence averages are obtainable from $4^{\text {th }}$-order accurate cell face averages; from this follows (2) the formulation and documentation of suitable discretizations for the gyrokinetic equation and the gyrokinetic Poisson equation; the gyrokinetic equation discretization is $4^{\text {th }}$ order multidimensional flux-corrected transport with hyperviscosity and limiting; the GK Poisson equation is discretized with a compact $4^{\text {th }}$ order stencil, and solved with a multigrid linear solver; $4^{\text {th }}$-order accuracy has been verified on test problems; the implementation of stencils for the GKPoisson equation in the Hypre library is underway (3) a suite of test problems for the hyperbolic integrator has been specified; implementation and testing are underway; (4) support for mapped grids has been added to Chombo, and tested on model mappings. The goal is a coupled gyrokinetic equation/electrostatic field-solve prototype by the end of the calendar year.

\section{Recent publications}

(1) X.Q. Xu, K. Bodi, J. Candy, B. I. Cohen, R. H. Cohen, P. Colella, M. R. Dorr, J. A. Hittinger, G. D. Kerbel, S. Krasheninnikov, W. M. Nevins, H. Qin, T. D. Rognlien, P. B. Snyder, M. V. Umansky, Z. Xiong, "Continuum Edge Gyrokinetic Theory and Simulations", in Fusion Energy 2006 (Proc. 21 st Int. Conf. Chengdu, 2006) (Vienna: IAEA) CD-ROM file TH/P6-23 and http://wwwnaweb. iaea.org/napc/physics/FEC/FEC2006/html/index.htm

(2) H. Qin, R.H. Cohen, W.M. Nevins and X.Q. Xu, Contrib. Plasma Phys., 46, 477 (2006)

(3) ) H. Qin, R.H. Cohen, W.M. Nevins and X.Q. Xu, Phys. Plasmas, 14, 056110 (2007).

(4) X.Q. Xu , Z. Xiong, M.R. Dorr, J.A. Hittinger, et al., "Edge Gyrokinetic Theory and Continuum Simulations", accepted by Nuclear Fusion.

(5) Z.Xiong, R. Cohen, T. Rognlien and X. Xu, "A high-order finite-volume algorithm for Fokker-Planck collisions in magnetized plasmas", submitted to J. Comp. Phys.

(6) X.Q. Xu, Z. Xiong, W. Nevins and G. McKee, "TEMPEST simulations of collisionless damping of GAM and neoclassical residual in edge plasma pedestal", submitted to Phys. Rev. Lett.

(7) R.H.Cohen and X.Q. Xu, (2007), "Progress in Kinetic Simulation of Edge Plasmas", submitted to Contrib. Plasma Phys. 